\title{
A Case Study of Integer Sum Reduction using Atomics
}

\author{
Zheming Jin and Hal Finkel \\ Argonne National Laboratory \\ 9700 Cass Ave, Lemont, IL 60439 \\ zjin@anl.gov, hfinkel@anl.gov
}

\begin{abstract}
This paper presents the implementations of integer sum reduction using atomic functions on FPGA, CPU, and GPU platforms. We explain the implementations and optimizations of the kernel using an OpenCL-based highlevel synthesis flow for an FPGA. In addition, we describe the optimizations of the reduction using directives for a multi-core CPU and a GPU. The experimental results show that the reduction on an Nvidia $\mathrm{K} 80 \mathrm{GPU}$ is $3.4 \mathrm{X}$ and $6.7 \mathrm{X}$ faster than an Intel Xeon 16-core CPU and an Arria 10 GX1150 FPGA, respectively. However, the FPGA consumes $4.4 \mathrm{X}$ and 2.3X less power than the CPU and GPU, respectively. The performance per watt on the FPGA is 2.2X higher than that on the CPU and 2.9X lower than that on the GPU.
\end{abstract}

\section{INTRODUCTION}

Reduction is an important type of parallelism exploited in many applications that include map reduce applications, machine learning, numerical analysis, and scientific simulation applications. Although graphics processing units (GPUs) and/or central processing units (CPUs) are ubiquitously used for achieving high-performance reductions, there are few studies on a reduction kernel using atomic functions (atomics) in a high-level synthesis (HLS) flow for field-programmable gate arrays (FPGAs). Atomics facilitate fine-grained concurrency in which threads can execute without blocking other threads. Atomics have been demonstrated empirically to be a highly efficient synchronization method for conventional multiprocessors [1], but their support of Open Computing Language (OpenCL) standard for FPGAs is limited. Intel's FPGA software development kit (SDK) for OpenCL provides 32bit atomic operations, but the user guide warns that they could decrease kernel performance when there are different atomic operations in an OpenCL kernel [2]. Atomics are currently not supported by Xilinx's SDAccel SDK.

Publication rights licensed to ACM. ACM acknowledges that this contribution was authored or co-authored by an employee, contractor or affiliate of the United Stated government. As such, the United States Government retains a nonexclusive, royalty-free right to publish or reproduce this article, or to allow others to do so, for Government purposes only.

HEART 2018, June 20-22, 2018, Toronto, ON, Canada

(C) 2018 Copyright is held by the owner/author(s). Publication rights

licensed to ACM. ACM ISBN 978-1-4503-6542-0/18/06 . . \$15.00

DOI: https://dx.doi.org/10.1145/3241793.3241809
In this paper, we explore the implementations of the sum reduction using atomics on FPGA, CPU, and GPU computing platforms. We seek to better understand the characteristics of an atomic operation and its performance impact on these computing platforms. To that end, we describe the implementations and optimizations of the reduction using OpenCL atomics on an FPGA. Then we present the optimized implementations of the reduction using OpenMP and OpenACC directives. Using directives for accelerator programming is a productive and portable way to optimize a kernel in parallel computing. Then we measure the raw performance, power, and performance per watt of the reduction on an Arria10-based FPGA platform, an Intel Xeon 16-core CPU, and an Nvidia Tesla K80 GPU.

The experimental results show that fully utilizing the bandwidth of the data bus on the target FPGA system can improve the kernel performance by a factor of 14.4. Using local memories to store the partial reductions, we can achieve 6.7X performance speedup over the reduction with a global memory. When both optimizations are enabled, they can lead to $112 \mathrm{X}$ speedup. We observe that kernel duplication can further improve the performance by a factor of 2.9. Although the raw performance of the reduction on the FPGA is the lowest, the FPGA consumes $4.4 \mathrm{X}$ and 2.3X less power than the CPU and GPU, respectively. The performance per watt of the kernel implementation on the FPGA is $2.2 \mathrm{X}$ higher than that on the CPU and 2.9X lower than that on the GPU. The FPGA results based on a HLS flow are promising; FPGAs will become a major heterogeneous computing component for energy-efficient high-performance computing.

The rest of the paper is organized as follows. The background, which we will briefly present in Section 2, is mainly about the OpenCL essentials. Section 3 defines the scope of the reduction for the paper. Section 4 describes the implementations and optimizations of the kernel. The experimental results are presented in Section 5. Section 6 summarizes the studies on atomics using a high-level synthesis flow. Section 7 concludes the paper.

\section{BACKGROUND}

The traditional hardware development flow using hardware description languages (HDLs) bars a wider audience from evaluating applications on FPGA-based heterogeneous computing systems. Emerging HLS tools [3,4] could provide an alternative for the scientific computing community. As most researchers have little experience with FPGA-based hardware development, using high-level 
programming language, particularly OpenCL for FPGA, could allow them to take advantage of the potential benefits of FPGAs.

An OpenCL application consists of host and kernel programs. The host program is written in standard $\mathrm{C} / \mathrm{C}++$ that runs on most of modern microprocessors. A host program needs to manage a set of OpenCL-related resources that facilitate kernel execution. A kernel is a function declared in a program and executes on an OpenCL-enabled device such as a GPU or an FPGA. Kernels can be executed in multiple instances. Each instance is defined as a work-item. These work-items are organized into multiple work-groups, and can be identified in one, two or three dimensions referred to as the $\mathrm{N}$ Dimension Range (NDRange). A work-item is executed as part of a work-group executing on a compute unit that comprises processing elements and memory interfaces. The

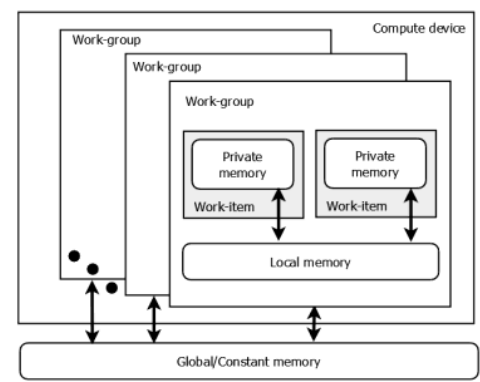

Figure 1. Memory model in OpenCL

execution order of different workgroups within a kernel is unsynchronized. A user can use explicit barriers to ensure the memory consistency for work-items within a workgroup. All the work-items belonging to that work-group must reach it before any of them can proceed beyond it. In addition, OpenCL defines a four-level memory hierarchy as shown in Figure 1.

\section{SUM REDUCTION}

Sum reduction is a common data parallel primitive. Listing 1 shows the sequential integer sum reduction in our study. The integer operations can be done in any order due to the associativity of the integer add. We can take advantage of associativity to divide the reduction into independent partial sums, and then combine results from the partial sums. This can be generalized to reductions on vectors of arbitrary size. This paper is focused on the unsegmented form of the sum reduction. It takes a binary associative operator "+" and an array of $M$ data as inputs, and returns as output one value.

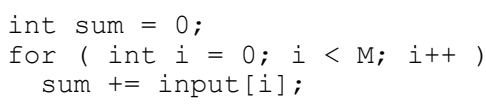

Listing 1. The sequential sum reduction
Sum reduction can be realized sequentially or in parallel. For the parallel reduction, we assume that the array length is large enough that is unfit for a single work-group. To reduce over large vectors, there are three general ways described in [5]. We choose atomic operations for reductions to evaluate the performance of reduction primitive on heterogeneous computing platforms. Atomic operations are useful in safely updating a shared numeric variable by multiple threads, such as on hardware platforms that support atomic operation. They are also useful in implementing barrier synchronization within a kernel [6], constructing complex data structures such as hash tables [7] or building high-level programming frameworks such as MapReduce [8]. The details of the atomic function are described in $[9,10]$. In this paper, the 64-bit sum reduction is beyond the scope of the paper because the 64-bit atomic functions are not supported in current HLS tools.

\section{IMPLEMENTATIONS OF THE REDUCTION IN OPENCL}

\subsection{Baseline}

The baseline reduction kernel B1 is shown in Listing 2 . Each work-item reads a unique element from the input vector in the global memory space implied by the global qualifier. The restrict keyword is recommended for each global address space to prevent the compiler from creating unnecessary memory dependencies between nonconflicting memory accesses. The OpenCL API function atomic_add() performs an atomic addition of input data to the content of memory. The kernel is an NDRange kernel with the global work size equal to the length of the input vector.

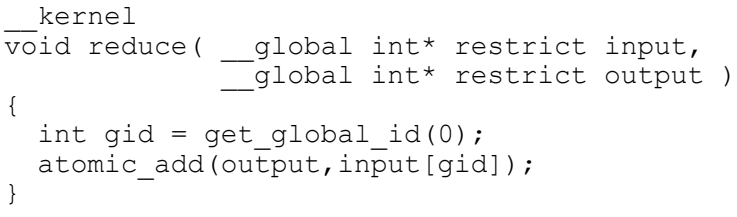

Listing 2. The baseline reduction kernel B1

An alternative kernel B2 is shown in Listing 3. Compared to B1, we explicitly use the identifiers (IDs) of a workgroup and a work-item in a work-group to index the input array. The identifiers and the size of a work-group (WGS) are queried with the OpenCL API functions.

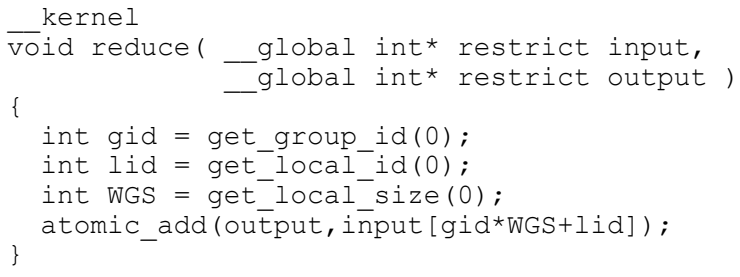

Listing 3. An alternative OpenCL reduction kernel B2

\subsection{Optimizations of Memory Accesses}

On our target FPGA platform, the data width between the DDR memory controller and the user FPGA design is 512- 
bit. To fully utilize the bandwidth of the data bus, we make use of the OpenCL API function vload16 to read 16 integers as a vector for each memory transaction. Listing 4 shows the kernel B3. It reads 16 integers as a vector from the global memory for each load, and then atomically adds the sum of the integers to the output.

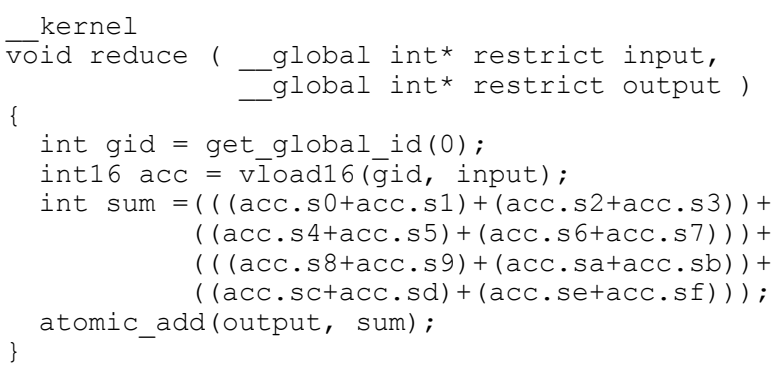

Listing 4. Kernel B3 loads 16 integers as a vector

Current FPGAs provide a large amount of on-chip memories. For example, Intel's Arria 10 series have approximately 8 Mbytes of internal memory. To reduce the overhead of memory accesses from the external memory, a local memory can be statically allocated for reusing the data. A local memory corresponds to an on-chip memory that utilizes the Block RAMs (BRAMs) in FPGAs. The BRAM blocks are configurable memory modules, and they are physically located near the kernel computation compared to the global memory. They allow single-cycle random memory accesses, thus significantly improving memory access performance.

Listing 5 shows the kernel B4 that utilizes a local memory for storing partial sum results. The first work-item in each work-group resets the local sum. Then a barrier synchronizes the work-items to use the initialized local sum. After the atomic add is performed by all work-items in a work-group, the last work-item in the group atomically adds the partial sum to the output. As mentioned in [2], the default maximum work-group size is 256 when a kernel contains a barrier.

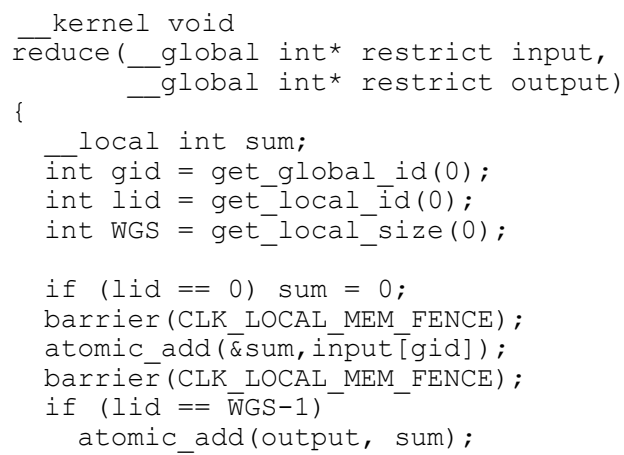

Listing 5. Kernel B4 performs atomics over local memory

\subsection{Reduction with Local Memories}

So far we have performed atomic operations over a single variable. Could we mitigate the pressure of atomic operations on a single memory by distributing the operations over multiple memory locations?
Listing 6 shows the generalized kernel using multiple local memories. The variable "N" represents the number of local memory locations. The modulo operator intends to distribute the atomic adds evenly for the $N$ memory locations among all the work-items. After all partial sums are computed atomically, they are accumulated to compute atomically the final sum. Furthermore, the kernel fully utilizes the data bandwidth between the memory controller and user logics.

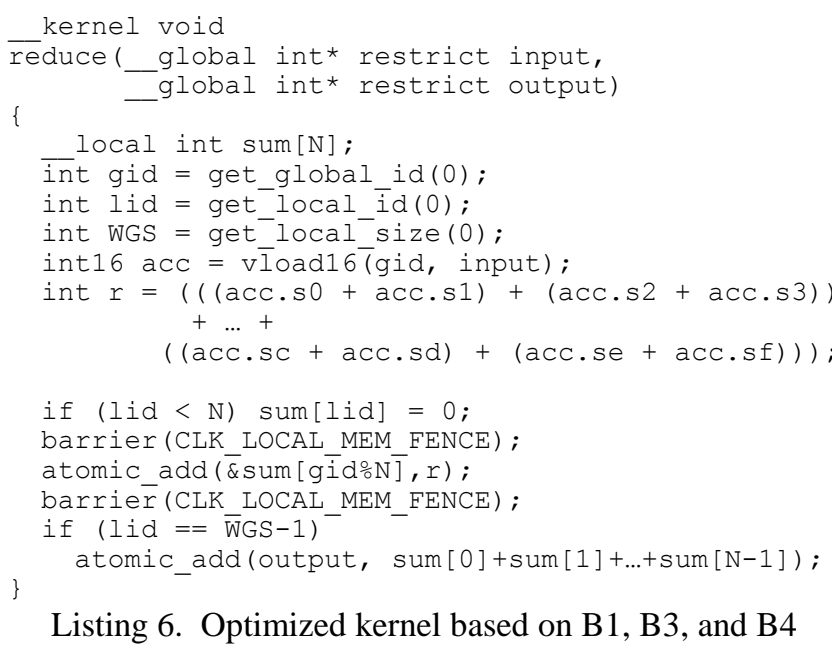

\subsection{Compute Unit Duplication}

Compute unit replication (kernel duplication) is an approach to increasing kernel performance by instantiating multiple compute units for each kernel. Each compute unit has its own memory access interface for the kernel function. Each compute unit is capable of executing multiple workgroups simultaneously to increase data throughput. However, multiplying the number of compute units increases global memory bandwidth contention among compute units, which can degrade the performance of memory-bound kernels that have non-streaming memory access patterns.

\section{EXPERIMENTS}

\subsection{Experimental Setup}

In this study, we used Nallatech 385A FPGA card that features an Arria 10 GX1150 FPGA chip and two 4GB DDR3L-2133 memory channels. The 385A card is installed on a host machine using a PCIe $x 83.0$ interface. The theoretical DDR3 memory bandwidth of the card is approximately $34 \mathrm{~GB} / \mathrm{s}$. The kernel execution time is measured with the OpenCL API function clGetEventProfilingInfo that returns the time in nano-seconds consumed by the kernel execution on the hardware. In our experiment, the length of the input vector ranges from $2^{9}(512)$ to $2^{27}$ (128 million) integers. Table 1 lists the characteristics of the OpenCL kernels in our experiment. When vload1 6 is used in a kernel, the global work size is divided by 16 as each work-item loads 16 input integers from global memory. The lower bound to the work- 
Table 1. Characteristics of OpenCL reduction kernels

\begin{tabular}{llll}
\hline $\begin{array}{l}\text { Kernel } \\
\text { name }\end{array}$ & $\begin{array}{l}\text { Work-group } \\
\text { size range }\end{array}$ & $\begin{array}{l}\text { Vector } \\
\text { load width }\end{array}$ & $\begin{array}{l}\text { Local } \\
\text { memory size }\end{array}$ \\
\hline B1 & {$\left[1,2^{27}\right]$} & 1 & 0 \\
\hline B2 & {$\left[1,2^{27}\right]$} & 1 & 0 \\
\hline B3 & {$\left[1,2^{23}\right]$} & 16 & 0 \\
\hline B4 & {$[1,256]$} & 1 & 1 \\
\hline B5 & {$[8,256]$} & 1 & 8 \\
\hline B6 & {$[16,256]$} & 1 & 16 \\
\hline B7 & {$[1,256]$} & 16 & 1 \\
\hline B8 & {$[8,256]$} & 16 & 8 \\
\hline B9 & {$[16,256]$} & 16 & 16 \\
\hline
\end{tabular}

group size of the kernels (B3 to B9) is constrained by the number of local memories. The work-group size, which must be a power of two, is a variable that will be evaluated in our experiment.

\subsection{Resource Usage of the Implementations}

The resource utilizations for all the kernels except kernel duplication described in the last section are almost the same. The logic utilization is $12 \%$, and the number of RAM blocks increase from 307 to 329 due to the increase of local memories in the kernels. The dedicated arithmetic processing unit (DSP) is not utilized by any kernels. When the kernel is duplicated by a factor of eight, the logic utilization increases from $12 \%$ to $17 \%$, and the number of RAM blocks is 547 .

\subsection{Kernel Performance on the FPGA}

Figure 2 shows the execution time of the two baseline kernels with respect to the work-group size using an array size of $2^{27}$. When the input array is indexed with the global ID in B1, the kernel time stays at approximately $6229 \mathrm{~ms}$, insensitive to the work-group sizes. However, the workgroup size has a large impact upon the performance of B2. The execution time decreases by a factor of 4 when the work-group size increases from 1 to 512 . When the size is 512 , the execution time of $\mathrm{B} 2$ is $4.6 \%$ slower than $\mathrm{B} 1$. The execution time of B2 levels off at $6519 \mathrm{~ms}$ when the work-

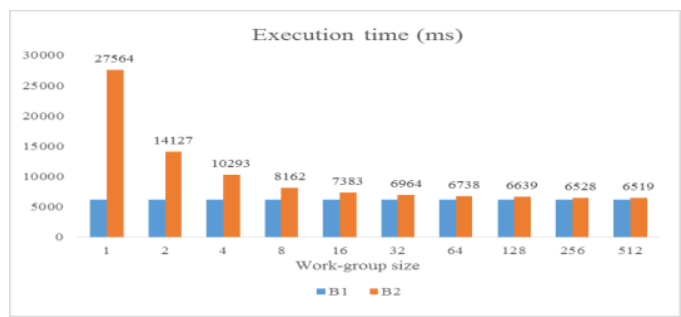

Figure 2. Performance of the two baseline kernels group size is larger than 512. The result suggests that the application does not effectively hide the overhead of the memory address generation using explicit work-group size and local ID until a work-group size of 64 or more. As the performance of B1 is better than B2 across the work-group sizes in our experiment, we will evaluate the performance of other kernels that index the input vector using global IDs.

Figure 3 shows the execution time of the kernels (B4, B5, B6) that contain one, eight, and sixteen local memories, respectively. The bars in the graph shows that the lowest execution time is approximately $939 \mathrm{~ms}$ when the workgroup size is larger than or equal to 64 . On the other hand, the execution time starts to saturate for work-group sizes larger than 64. Compared to B1 that performs atomic operation over global memory, using local memory can reduce the execution time by a factor of 6.7. Figure 3 also reveals that using more local memories slightly increases the execution time from $939 \mathrm{~ms}(\mathrm{~N}=1)$ to $951 \mathrm{~ms}(\mathrm{~N}=16)$ due to the arbitration of local memory accesses.

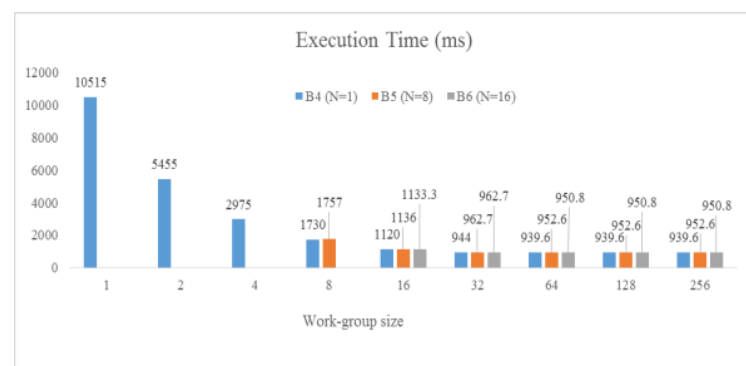

Figure 3. Performance of the kernel implementations with respect to work-group size and local memories.

Figure 4 shows the execution time of the four kernels (B3, B7, B8, B9) that fully utilize the 512-bit data bus. The kernels (B7, B8, B9) contain one, eight, and sixteen local memories for atomic operations, respectively. Compared to B1, the execution time of B3 stays at around $433 \mathrm{~ms}$ for different work-group sizes, achieving $14.4 \mathrm{X}$ reduction in execution time. On the other hand, the combination of using local memory and vectorized load can lower the execution time by a factor of 16.2 and 7.4 respectively compared to each individual optimization. The results show the

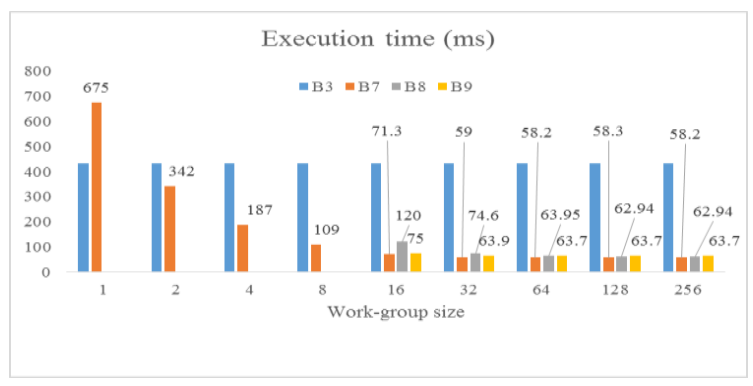

Figure 4. Performance of the kernel implementations with respect to work-group sizes and local memories. Each kernel reads 16 integers as a vector. 
compute time of the two kernel implementations when the input array sizes range from $2^{9}$ to $2^{27}$. The number of local sums is 256. The $y$ axis is scaled logarithmically with a base of 2 while the $x$ axis only shows the exponent. The threshold is $2^{21}$, above which $\mathrm{v} 2$ is better than $\mathrm{v} 1$. When the array size is $2^{27}$, the lowest time of $\mathrm{v} 2$ is $2.957 \mathrm{~ms}$, approximately $1.87 \mathrm{X}$ faster than $\mathrm{v} 1$.

To compare the performance, power, and performance per watt on the three platforms, we choose the implementations that achieve the highest performance for the largest array size on the three platforms. Figure 7 shows that the reduction on the GPU takes approximately $2.957 \mathrm{~ms}, 3.4 \mathrm{X}$ and 6.7X faster than the CPU and FPGA, respectively. However, the FPGA consumes approximately $30 \mathrm{~W}, 4.4 \mathrm{X}$ and 2.3X lower than the CPU and GPU, respectively. The performance per watt, defined as the million number of array elements that are reduced per second per watt, is 223.7 on the FPGA, 2.2X higher than that on the CPU and 2.9X lower than that on the GPU. There are a few factors that lead to the low FPGA performance. The kernel is memory-bound and the theoretical peak memory bandwidth on the FPGA is $3.5 \mathrm{X}$ and $7.05 \mathrm{X}$ lower than that on the CPU and GPU, respectively. The work-items on an FPGA are executed in a pipeline to save the limited memory bandwidth, which is different from the execution of workitems on a GPU where they are processed in a data parallel manner. The upper bound to the kernel clock frequency on the FPGA is around $300 \mathrm{MHz}$ compared to $562 \mathrm{MHz}$ on the GPU and $3.4 \mathrm{GHz}$ on the CPU.

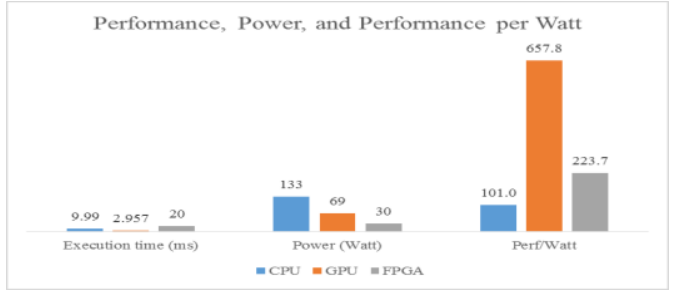

Figure 7. Performance, power, and performance per watt on the CPU, GPU, and FPGA.

\section{RELATED WORK}

There are extensive studies on the performance characterizations of reductions and atomic functions on GPUs $[12,13,14,15,16]$. However, understanding the performance of reduction using atomic functions on an OpenCL FPGA platform is important as FPGAs are becoming promising heterogeneous computing components for HPC. Early research was focused on maximizing the floating-point performance under the constraint of memory bandwidth and resource usage using HDL [17]. Recently, the author evaluated an integer reduction kernel using OpenCL on a Xilinx ZYNQ FPGA board [18]. They did not fully utilize the data bus on the target FPGA platform, and implemented the segmented version of reduction. It was reported that atomics are 'expensive' when implementing locks in their study of data partition problem within an FPGA [19]. However, the authors applied atomics to the OpenCL implementation of a K-means clustering algorithm and achieved 1.5X-1.9X speedup [20]. In this paper, we are focused on the performance of the atomics and its performance implications on heterogeneous platforms.

\section{CONCLUSION}

The experimental results show that current HLS tools do not take advantage of platform-specific optimizations automatically; simple porting would not guarantee performance portability. Although there is performance and power tradeoff when using atomics, they can simplify a kernel design, and allow the creation of more sophisticated algorithms. They also promote productivity and program portability. Hence, it will be worthwhile for HLS tools to adopt atomics and enhance their functionality, performance, and verification.

\section{ACKNOWLEDGMENTS}

The research work was supported by the U.S. Department of Energy under contract DE-AC02-06CH11357.

\section{REFERENCES}

[1] Gramoli, V., 2015, January. More than you ever wanted to know about synchronization: synchrobench, measuring the impact of the synchronization on concurrent algorithms. In ACM SIGPLAN Notices (Vol. 50, No. 8, pp. 1-10). ACM.

[2] Intel FPGA SDK for OpenCL Programming Guide. UG-OCL002. 2017.05.08

[3] Nane, R., Sima, V.M., Pilato, C., Choi, J., Fort, B., Canis, A., Chen, Y.T., Hsiao, H., Brown, S., Ferrandi, F. and Anderson, J., 2016. A survey and evaluation of fpga high-level synthesis tools. IEEE Transactions on Computer-Aided Design of Integrated Circuits and Systems, 35(10), pp.1591-1604.

[4] Koch, D., Hannig, F. and Ziener, D. eds., 2016. FPGAs for Software Programmers. Springer.

[5] https://developer.amd.com/resources/articles-whitepapers/opencloptimization-case-study-simple-reductions/

[6] Xiao, S. and Feng, W.C., 2010, April. Inter-block GPU communication via fast barrier synchronization. In Parallel \& Distributed Processing (IPDPS), 2010 IEEE International Symposium on (pp. 1-12). IEEE.

[7] Michael, M.M., 2002, August. High performance dynamic lock-free hash tables and list-based sets. In Proceedings of the fourteenth annual ACM symposium on Parallel algorithms and architectures (pp. 73-82). ACM.

[8] Hong, C., Chen, D., Chen, W., Zheng, W. and Lin, H., 2010, September. MapCG: writing parallel program portable between CPU and GPU. In Proceedings of the 19th international conference on Parallel architectures and compilation techniques (pp. 217-226). ACM.

[9] https://software.intel.com/en-us/articles/using-opencl-20-atomics

[10] Khronos OpenCL Working Group, The OpenCL Specification, version 1.2, 15 November 2011. Cited on pages, 18(7), p.30

[11] Kirk, D.B. and Wen-Mei, W.H., 2016. Programming massively parallel processors: a hands-on approach. Morgan Kaufmann.

[12] Mark, H., 2008. Optimizing parallel reduction in CUDA. NVIDIA CUDA SDK.

[13] https://developer.amd.com/resources/articles-whitepapers/opencloptimization-case-study-simple-reductions/

[14] Martín, P.J., Ayuso, L.F., Torres, R. and Gavilanes, A., 2012, July. Algorithmic strategies for optimizing the parallel reduction primitive in CUDA. In High Performance Computing and Simulation (HPCS), 2012 International Conference on (pp. 511-519). IEEE.

[15] Egielski, I.J., Huang, J. and Zhang, E.Z., 2015. Massive atomics for massive parallelism on GPUs. ACM SIGPLAN Notices, 49(11), pp.93-103. 
[16] Elteir, M., Lin, H. and Feng, W.C., 2011, September. Performance characterization and optimization of atomic operations on amd gpus. In Cluster Computing (CLUSTER), 2011 IEEE International

Conference on (pp. 234-243). IEEE.

[17] Zhuo, L., Morris, G.R. and Prasanna, V.K., 2005, April. Designing scalable FPGA-based reduction circuits using pipelined floatingpoint cores. In Parallel and Distributed Processing Symposium, 2005. Proceedings. 19th IEEE International (pp. 8-pp). IEEE.

[18] http://svenssonjoel.github.io/writing/zynqreduce.pdf

[19] Wang, Z., He, B. and Zhang, W., 2015, September. A study of data partitioning on OpenCL-based FPGAs. In Field Programmable Logic and Applications (FPL), 2015 25th International Conference on (pp. 1-8). IEEE.

[20] Ramanathan, N., Wickerson, J., Winterstein, F. and Constantinides, G.A., 2016, February. A case for work-stealing on FPGAs with OpenCL atomics. In Proceedings of the 2016 ACM/SIGDA International Symposium on Field-Programmable Gate Arrays (pp. 48-53). ACM 